\title{
Analyzing Heterochromatin Formation Using Chromosome 4 of Drosophila melanogaster
}

\author{
K.A. Haynes, B.A. Leibovitch, S.H. Rangwala, C. Craig, and S.C.R. Elgin \\ Department of Biology, Washington University, St. Louis, Missouri 63130
}

While chromosomes provide a broad level of genomic organization within the nuclei of higher eukaryotes, the chromosome itself is further organized into multiple domains with distinct properties. This level of organization is visible in interphase nuclei as areas of condensed heterochromatin and regions of more dispersed euchromatin. Chromatin domains are biochemically distinguished by the types of histone modification and associated nonhistone chromosomal proteins. In most higher eukaryotes, domains of constitutive heterochromatin are normally restricted to pericentric and telomeric DNA. A remarkable property of heterochromatin in fungi, flies, and mammals is the ability to spread in cis, in response to loss of boundary constraints or to changes in dosage or activity of chromatin components; this results in silencing of euchromatic genes that are abnormally juxtaposed to heterochromatic domains by chromosome rearrangement or transposition, referred to as Position Effect Variegation (PEV) (for review, see Grewal and Elgin 2002). Recent studies in fungi and plants suggest that heterochromatin formation is targeted to repetitious elements through an RNAi mechanism, resulting in a domain of silenced chromatin (Volpe et al. 2002; Matzke et al. 2004; Schramke and Allshire 2004).

The small fourth chromosome of Drosophila melanogaster exhibits a rather unusual chromatin organization compared to the other chromosome arms. The distal $20-25 \%$ of chromosome 4 is amplified in polytene nuclei to a similar extent as the other euchromatic arms, and contains 82 genes in $1.2 \mathrm{Mb}$ of DNA (Flybase Consortium 2003), a gene density comparable to that found in other euchromatic regions. At the same time, chromosome 4 exhibits characteristics of heterochromatin throughout its length. Chromosome 4 is rich in dispersed repetitious sequences (Kaminker et al. 2002), shows no detectable meiotic recombination (Bridges 1935), and is late-replicating (Barigozzi et al. 1966), all well-established characteristics of heterochromatic regions. Further, immunofluorescent staining of polytene chromosomes shows that Heterochromatin Protein 1 (HP1), known to play a key role in heterochromatin-induced silencing, is localized both in the pericentric heterochromatin and across the whole of the fourth chromosome (Fig. 1A) (James et al. 1989; Eissenberg and Elgin 2000).

\section{CHROMOSOME 4: INTERSPERSED HETEROCHROMATIC AND EUCHROMATIC DOMAINS DEMONSTRATED BY IMMUNOCYTOLOGY}

Unlike the fairly homogenous staining visible throughout the chromocenter, the fourth possesses contrasting

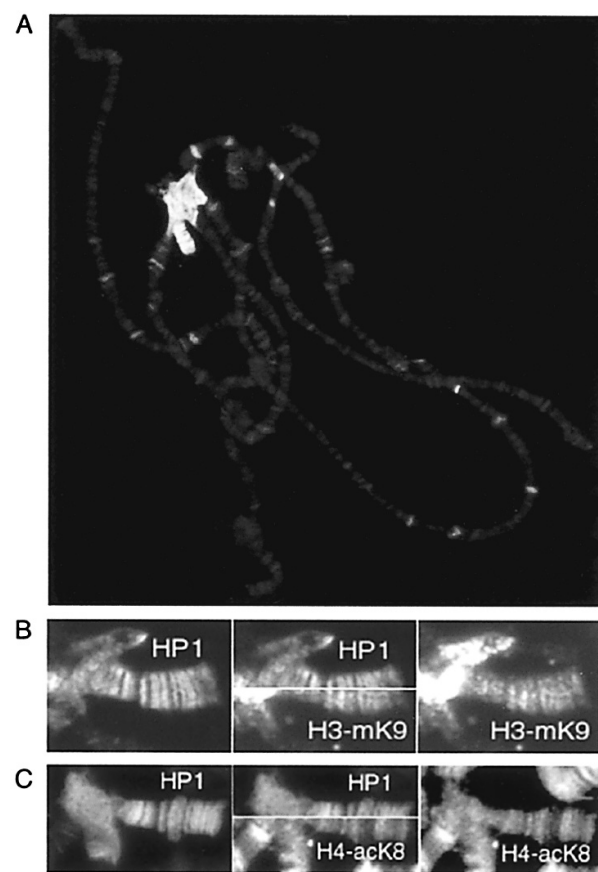

Figure 1. Immunofluorescent staining of polytene chromosomes of Drosophila melanogaster using antibodies specific for HP1 and antibodies specific for a modified histone. While the distribution pattern for histone $\mathrm{H} 3$ methylated at lysine 9 is essentially congruent with that for HP1, the distribution of histone $\mathrm{H} 4$ acetylated at lysine 8 is often opposing, suggesting distinct heterochromatic and euchromatic domains. $(A)$ An entire chromosome set; $(B, C)$ an enlargement of the fourth chromosome. (A) Immunofluorescent staining of polytene chromosomes from third instar larvae was carried out as described (James et al. 1989 ) using antibodies specific for HP1 (mouse monoclonal C1A9, James et al. 1989). (B) Chromosomes were stained simultaneously with anti-HP1 (mouse C1A9) and anti-H3-mK9 (rabbit, Upstate Biotechnology); secondary antibodies (Molecular Probes) were labeled with Alexa Fluor red or green. $(C)$ Chromosomes were stained simultaneously with anti-HP1 (mouse C1A9) and rabbit anti-H4-acK8 (Suka et al. 2001). 
HP1-rich and HP1-lacking bands, suggesting the presence of alternating domains of heterochromatin and euchromatin. This idea can be tested by examining the pattern of histone modification. Histone modifications have been shown to play a critical role in establishing alternative forms of chromatin packaging. Methylation of histone $\mathrm{H} 3$ at lysine 9 (producing $\mathrm{H} 3-\mathrm{mK} 9$ ) has been linked to HP1 interaction and heterochromatin formation, while acetylation of most lysines in the $\mathrm{H} 3$ and H4 tails is associated with gene activation (for review, see Berger 2002; Grewal and Elgin 2002). Immunocytology of polytene chromosomes indicates that the fourth chromosome includes domains with modifications indicative of both heterochromatin and euchromatin (Fig. 1). Distribution of the H3-mK9 isoform aligns with the distribution of HP1, as anticipated (Fig. 1B) (see also Schotta et al. 2002), while the $\mathrm{H} 3$-acK8 isoform has a distribution in opposition to that seen for HP1 (Fig. 1C). Thus, while the prominent association of $\mathrm{HP} 1$ and $\mathrm{H} 3-\mathrm{mK} 9$ indicates a high percentage of heterochromatin, there are interspersed domains within the fourth that exhibit modifications associated with euchromatin, suggesting a pattern in which the active genes are clustered, flanked by heterochromatic domains.

These observations illustrate the need to organize distinct domains within the context of a single chromosome. The interaction of HP1 both with H3-mK9 and with the H3 histone methyltransferases able to generate that modification suggests a model for maintenance and spreading of heterochromatin (for review, see Grewal and Elgin 2002). On binding to the modified histone H3-mK9, HP1 can recruit $\mathrm{H} 3$ methyltransferase, facilitating modification of adjacent nucleosomes. This model is supported by recent findings in Schizosaccharomyces pombe. Spreading of heterochromatin occurs upon removal of a putative boundary of the silent mating type domain; this requires the yeast homologs of HP1 and SU(VAR)3-9, an H3-K9 methyltransferase (Hall et al. 2002). In flies, both mutations in HP1 and in SU(VAR)3-9 result in suppression of $\mathrm{PEV}$, i.e., in loss of silencing at a variegating gene (Eissenberg et al. 1990; Schotta et al. 2002). Thus, the maintenance and spread of heterochromatin are explained as a cycle of biochemical interactions between a histone signal, a structural protein, and the enzyme that generates the signal. However, the question of how this heterochromatin formation is targeted to specific domains remains unanswered.

\section{INTERSPERSED HETEROCHROMATIC AND EUCHROMATIC DOMAINS REPORTED BY hsp 70-white}

We have investigated fourth chromosome domain organization using a transposable $P$ element $P[h s p 26-p t$, hsp 70-w] (Fig. 2) as a phenotypic probe. Earlier studies have shown that while this $P$ element in a euchromatic domain bestows a full red eye phenotype on a white mutant line, transposition into pericentric or telomeric heterochromatin results in variegated expression of white, reduced nuclease accessibility in the $h s p 26$ regulatory region, and a shift to a more regularly spaced nucleosome array across the transgene (Wallrath and Elgin 1995; Cry-

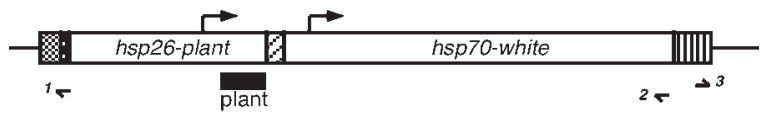

Figure 2. A map of the $P[h s p 26-p t, h s p 70-w]$ construct drawn to scale (Wallrath and Elgin 1995). The hsp26 sequences from -1917 to +490 were fused to a 740 -bp fragment of barley cDNA (plant probe), followed by $h s p 70$ transcription termination sequences (slashed box). This fusion gene was cloned into the $P$ element vector A412 possessing an $h s p 70$-driven copy of the white gene. Transcription start sites for hsp26-pt and hsp70-w are marked by bent arrows. (Left) The 3'P end (dotted box); (right) the 5' $P$ end (striped box). Primers used for inverse or direct PCR to identify flanking DNA (small arrows below the diagram) are 1 [5'-aactcgaggectcgaggt- $\left.3^{\prime}\right] ; 2$ [5'-gacgaaatgacc cactcgg-3']; 3 [5'-gcttcggctatcgacgggaccacc-3'].

derman et al. 1999a; Sun et al. 2001). Insertion of this element at various sites in the fourth chromosome has identified a few euchromatic domains (resulting in a red eye) interspersed with heterochromatic domains (causing a variegating eye) (Sun et al. 2000). Insertion sites have now been precisely mapped onto the published $D$. melanogaster genome sequence (Flybase Consortium 2003) using genomic DNA sequences flanking the $5^{\prime} P$ element end, recovered by inverse PCR. The distribution of insertion sites in lines from this study resulting in a variegating or red eye phenotype relative to the positions of genes and transposable elements is shown in Figure 3. The pattern of reporter gene expression reveals two euchromatic domains in a largely heterochromatic chromosome. The present map is most likely not saturated for euchromatic domains; in practice, it is much easier to recover fourth chromosome $P$ element insertion events from among variegating lines than from among red eye lines, as the bulk of the red eye lines represent insertion into the major euchromatic domains on the other chromosomes.

While one might anticipate that variegating inserts would be associated with gene-poor regions of the chromosome, potentially containing tandem arrays of repetitious elements, this proved not to be the case. In fact, most (eight of ten) of the variegating $P$ elements shown lie within $2 \mathrm{~kb}$ of a gene, five lying within the transcribed portions of four different genes. In all cases tested, the variegating phenotype is suppressed (loss of silencing) by a mutation in HP1 (Sun et al. 2000). Recently we have recovered and analyzed an additional 11 lines carrying the $P$ element on the fourth. The results support the conclusions discussed above, while identifying an additional euchromatic domain associated with the $s v$ gene (Sun et al. 2004).

Taken together, the banded pattern of HP1 distribution and the position-dependent expression of the $P$ element reporter inserted at different sites across the fourth chromosome strongly support a model of interspersed euchromatic and heterochromatic domains. Rather than finding the fourth chromosome genes restricted to euchromatic domains, the experimental results described above point to the conclusion that many fourth chromosome genes lie in heterochromatic domains, defined as regions inducing a variegating white phenotype. One can infer that a significant number of fourth chromosome genes are packaged with HP1. This is not without prece- 


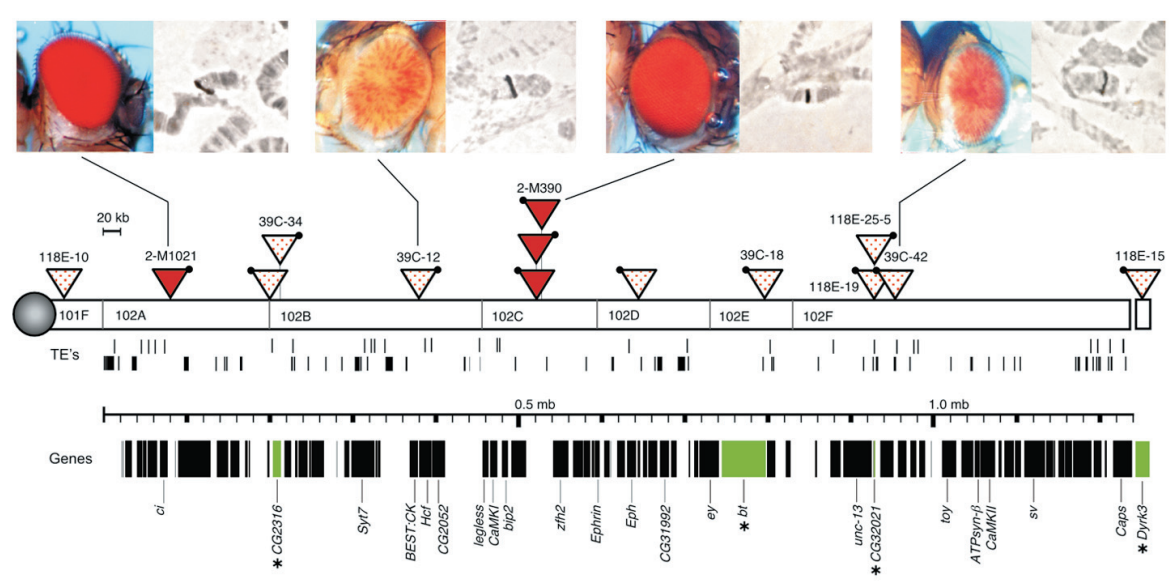

Figure 3. Chromosome 4 is made up of interspersed heterochromatic and euchromatic domains, as reported by hsp 70-white. Characteristics of representative $P$ insert lines are shown at the top: photographs of the eye phenotypes (left) are adjacent to photographs showing the cytological position of the $P$ element mapped by in situ hybridization of third instar larvae polytene chromosomes (right) for a given line. The map of the fourth chromosome (regions 101F-102F) shows the positions of known and predicted genes, and of transposable elements (TEs) (Flybase Consortium 2003). Solid triangles and dotted triangles mark $P$ element insertion sites that result in a solid red eye or induce a variegating phenotype, respectively. The black dot indicates the $5^{\prime}$ end of the $P$ element. In cases where a line has been recovered with a $P$ element inserted within a gene, the gene (in green) is indicated by an asterisk. Figure based on data from Sun et al. $(2000,2004)$.

dent. While much of the Drosophila heterochromatin at centromeres and telomeres is made up of tandem repeats, classical genetic analysis has identified several genes within the pericentric heterochromatin, and data from genome sequencing suggest that several hundred genes may reside in these regions in D. melanogaster (Hoskins et al. 2002). Many of the genes on the fourth have specific developmental functions. It will be of interest to determine how these genes function within a heterochromatic environment.

\section{THE SPATIAL POSITION OF CHROMOSOME 4, CLOSE TO PERICENTRIC HETEROCHROMATIN, IS CRITICAL TO MAINTAIN SILENCING}

Many lines of evidence from studies in yeast, flies, and mammals (for review, see Gasser 2001) demonstrate a link between gene silencing and nuclear location. We have used X-ray-induced translocation to show that the position of the $P$ element reporter in the nucleus, in addition to its position in the genome, can impact chromatin packaging. Transgenes that map to the distal portion of the fourth chromosome, such as that present in line 118E15 (see Fig. 3), show a variegating phenotype with similar responses to genetic modifiers as observed for the pericentric and other variegating fourth chromosome transgenes, including a loss of silencing on depletion of HP1 (Wallrath and Elgin 1995). By using X-ray mutagenesis we can generate translocations in line $118 \mathrm{E}-15$ to address whether nuclear position influences silencing (Cryderman et al. 1999b). Translocations resulting in a displacement of the fourth further away from the centromeric heterochromatin (stocks X-2 and X-4) lead to a dramatic loss of silencing (Fig. 4). However, when a translocation places the fourth in a new locus proximal to the pericentric heterochromatin (stock X-10), silencing is maintained. Variegation in all these cases is suppressed by $S u(v a r) 2-5^{02}$, a mutation in the gene encoding HP1. These results indicate that the local chromatin structure is impacted by changes in nuclear organization and suggest that the high concentration of HP1 in the pericentromeric heterochromatin may generate a local nuclear environment that facilitates heterochromatin formation and maintenance.

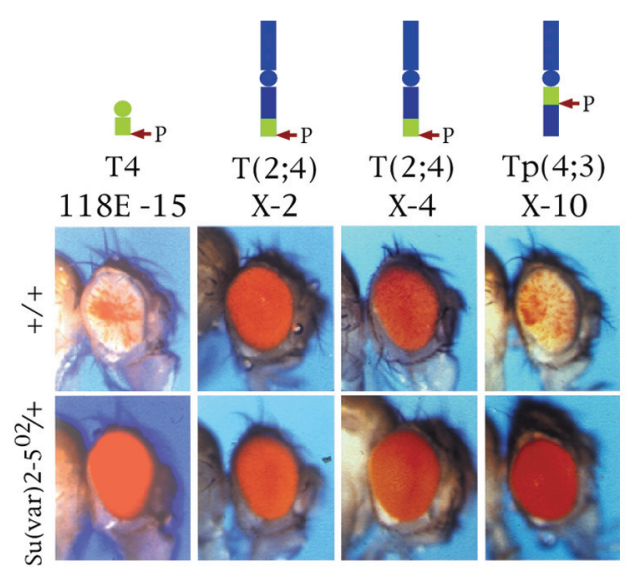

Figure 4. The spatial position of chromosome 4 is critical to maintain silencing. Diagrams of the relevant chromosomes (the fourth highlighted in green, second and third in blue) are shown at the top; a red arrow labeled "P" marks the $P$ element insertion $118 \mathrm{E}-15$ (see Fig. 3). Translocation of $\sim 1 \mathrm{Mb}$ of the distal portion of the fourth chromosome (carrying the $P$ element close to its telomere) to the distal end of chromosome 2 [stocks labeled $\mathrm{T}(2 ; 4)$ ] causes a loss of silencing of the white reporter. Transposition of approximately the same fourth chromosome fragment to a proximal region of the third chromosome [stock $\mathrm{Tp}(4 ; 3)]$ allows silencing. Variegation observed in these cases is suppressed by a mutation in the gene encoding HP1, Su(var)2-5 (Reprinted, with permission, from Cryderman et al. 1999b [@Nature Publishing Group] [http://www.nature.com/].) 


\section{HETEROCHROMATIC SILENCING, H3-K9 METHYLATION, AND HP1 LOCALIZATION IN DROSOPHILA ARE DEPENDENT UPON THE RNAi MACHINERY}

The presence of euchromatic domains on chromosome 4 argues against the simple idea that proximity to pericentromeric heterochromatin is sufficient to induce formation of heterochromatin, and suggests that cis-acting DNA sequence elements are required to target heterochromatin formation in specific regions. Repetitious elements have been implicated as targeted sites for heterochromatin formation in fungi and plants via an RNAi mechanism (Volpe et al. 2002; Hall et al. 2002; for reviews, see Matzke et al. 2004; Schramke and Allshire 2004). Region 101F-102F of chromosome 4 is enriched in repetitious sequences (see Fig. 3) compared to similar intervals on the other euchromatic chromosome arms (Kaminker et al. 2002). The average transposable element density is $10-15 / \mathrm{Mb}$ in the major chromosome arms, but $<82 / \mathrm{Mb}$ for chromosome 4 , because of an order-of-magnitude increase in remnants of LINE-like and TIR elements (elements that transpose via a DNA intermediate, flanked by short inverted repeats). A recent effort to generate a developmental profile of small RNAs from $D$. melanogaster has yielded a cloned and characterized nonredundant collection of 62 miRNAs and 178 repeat-associated small interfering RNAs (rasiRNAs). rasiRNAs were recovered from 38 different transposable elements (corresponding to $40 \%$ of the known elements), as well as from satellite DNA and the subterminal minisatellite at the $2 \mathrm{~L}$ telomere. Of these, 15 are represented in the $1.2-\mathrm{Mb}$ banded portion of chromosome 4 . The rasiRNAs, 16-28 nucleotides, were most abundant in testis and early embryo, the latter suggesting a role in establishing heterochromatin structure (Aravin et al. 2003).

To examine whether heterochromatin assembly in Drosophila might be targeted by siRNA, we tested the potential of available mutations in RNAi components piwi, aubergine, and homeless to impact heterochromatic silencing using two test systems, tandem mini-white arrays and white transgenes in either pericentric heterochromatin or a heterochromatin domain on the fourth chromosome. In both test systems, the white reporter gene shows a variegating phenotype that is suppressed (loss of silencing) in the presence of mutations in HP1 and exhibits other characteristics typical of heterochromatin-induced silencing. Both systems exhibited a loss of silencing (suppression of PEV) in the presence of mutations in the RNAi components (Fig. 5). The loss of silencing is accompanied by a decrease in the amount of H3-mK9 (in homeless mutant flies, to ca. 25\% that observed without the mutation) and by a redistribution of HP1 to sites within the euchromatic arms (Pal-Bhadra et al. 2004).

\section{DOES RNAI TARGET HISTONE MODIFICATION OR HP1 INTERACTIONS?}

A major question that remains is the connecting link between RNAi and heterochromatin formation in

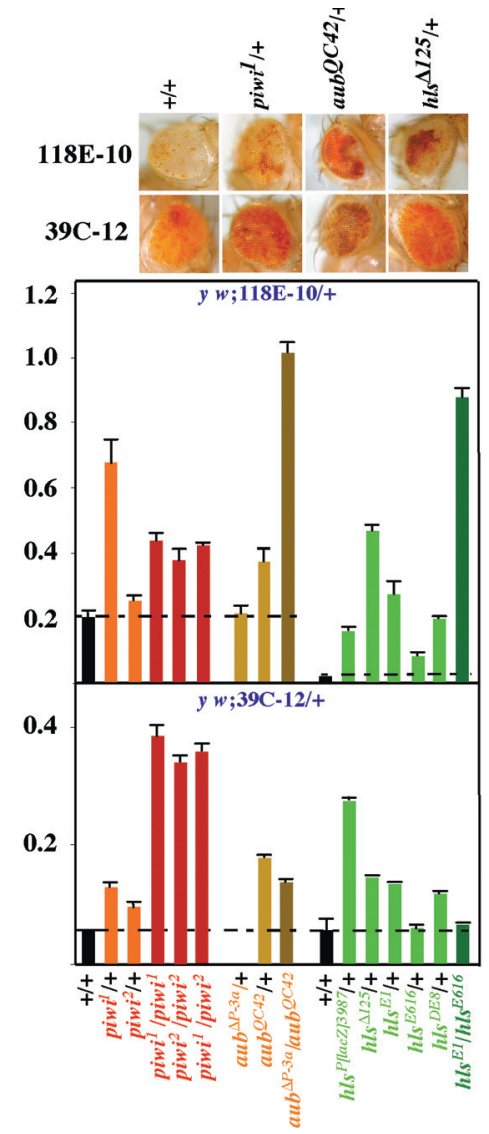

Figure 5. Loss of silencing is associated with mutations in components of the RNAi system. Homozygous or heteroallelic mutations in piwi result in an increase in white gene expression (loss of silencing) in line 118E-10 (white transgene in pericentric heterochromatin). The heteroallelic mutant combination of aubergine produces strong suppression. The homeless mutations have a dominant phenotype with a several-fold increase in expression, depending on the allele. Similar, but less dramatic results were obtained using stock 39C-12 (transgene in the fourth chromosome). Eye phenotypes are shown in photographs above. Pigment values (below) are given relative to the control $y w^{67 c 23}$ stock carrying the respective transgene. (Adapted, with permission, from Pal-Bhadra et al. 2004 [@AAAS].)

Drosophila. The recent characterization of the RITS complex (RNA-induced initiation of transcriptional gene silencing) in $S$. pombe provides some insight into this question. RITS is composed of siRNA (22-25 nucleotides); Chp1, a chromodomain protein that binds to centromeric repeats; Ago1, the fission yeast Argonaute homolog; and Tas3, a novel protein (Verdel et al. 2004). Gene silencing, H3-K9 methylation, and Swi6 (HP1) localization are dependent upon the function of all three RITS components.

Is there a similar targeting complex in Drosophila? A speculative model for targeting heterochromatin formation is presented in Figure 6. The model is based on the reported work in S. pombe, our studies in flies, work by others describing the components of the RNAi system (particularly the RISC complex) in Drosophila (for re- 
Figure 6. A speculative model of targeted heterochromatin formation. Long dsRNA is processed by the Dicer/R2D2 complex. The resulting siRNA (probably in a singlestranded form) may be complexed with RISC or another loading complex. We postulate a targeting complex that includes an siRNA from repetitious DNA, a chromo-domain protein (arguably HP1), and a PAZ/PIWI group member (possibly AUB or PIWI); HLS plays a critical role, but its placement is uncertain. At the heterochromatin initiation site, the targeting complex presumably interacts with a histone-modification complex, likely including HDAC1/ SU(VAR)3-9. We hypothesize that siRNA-dependent targeting of HP1 is the primary event, followed by histone modification to stabilize and maintain HP1 association (thick arrows). Alternative pathways are indicated by thin arrows.

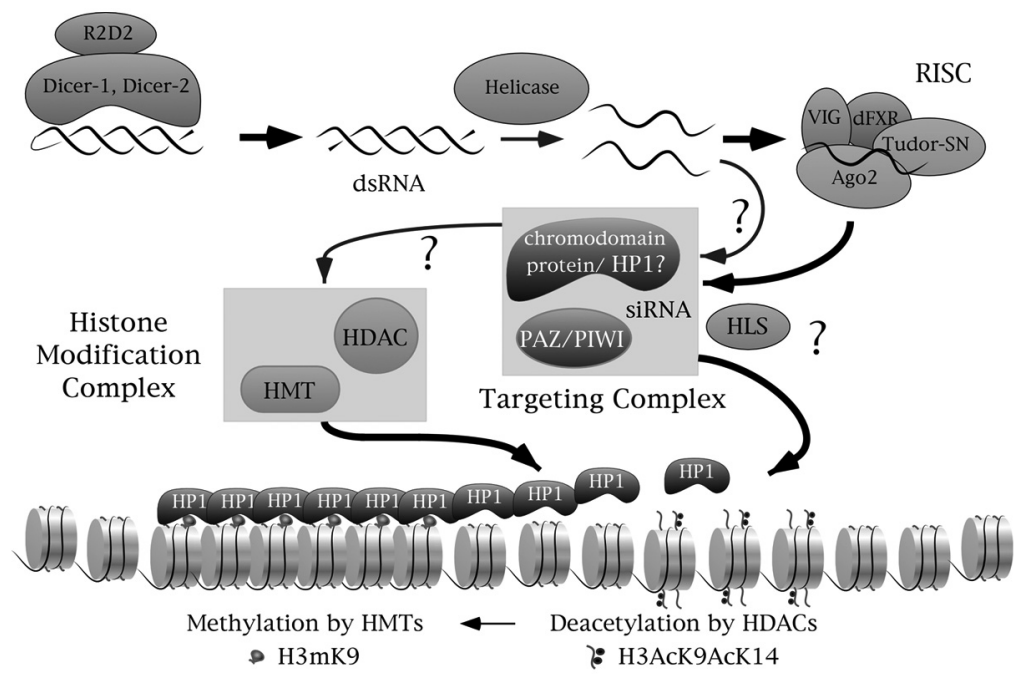

view, see Hannon 2002), and work describing histonemodification enzymes and heterochromatin complexes in Drosophila (for review, see Grewal and Elgin 2002). The model points to a number of important questions that need to be resolved. Is the RISC complex, known to be critical for posttranscriptional gene silencing, a required component for transcriptional gene silencing? What role does the homeless product play? Can one identify a "targeting complex" similar to RITS? While Drosophila has several members of the PAZ/PIWI family of proteins (AGO1, AGO2, PIWI, AUB), there are no homologs for Chp1 (aside from HP1) or Tas3. homeless activity is critical for proper localization of HP1 (Pal-Bhadra et al. 2004) and encodes a protein containing structural motifs that suggest the capacity to bind nucleic acids, to unwind double helices, and to interact with methylated peptides (Flybase Consortium 2003; Maurer-Stroh et al. 2003). We propose that HLS plays a critical role in the processing of siRNAs involved in transcriptional gene silencing, perhaps facilitating their interaction with RISC or perhaps participating directly in a targeting complex.

What sort of target might be present on the fourth chromosome? As noted above, the fourth chromosome is particularly rich in repetitious sequences, mostly fragments of transposable elements. We have used a genetic approach to search for cis-acting DNA sequences that specify heterochromatic domains. Genetic screens for a switch in phenotype on mobilization of the $P$ element reporter described above have demonstrated that local deletions or duplications of 5-80 kb of DNA flanking the transposon reporter can lead to loss or acquisition of variegation, pointing to short-range $c i s$-acting determinants of silencing. We have mapped the distance from the $P$ element to the surrounding repetitious DNA fragments for a series of lines derived from 39C-12 (see Fig. 3), inserted close to the $H c f$ gene in region 102B. The results suggest that if the $P$ element with $h s p 70-w$ lies within $10 \mathrm{~kb}$ of a fragment of the 1360 repetitious element, it will have a variegating phenotype, indicating heterochromatic packaging, whereas if it lies farther away, it will have a uniform red eye, indicating euchromatic packaging (Sun et al. 2004). This suggests that 1360 , and perhaps other repetitious elements present in D. melanogaster, could serve as the source of the siRNA in the putative Targeting Complex. Once targeted, heterochromatin formation apparently spreads over a distance of about $10 \mathrm{~kb}$, a result similar to that found in $S$. pombe at the mating type locus (Hall et al. 2002). The results to date do not identify any discrete boundaries to the spreading of HP1-dependent silencing in this case, but rather suggest a competition based on alternative histone modifications to establish the mode of chromatin packaging (Sun et al. 2004).

Once a locus is targeted for silencing via the RNAi pathway, how is heterochromatin packaging nucleated? Localization of HP1 and the histone-modification machinery appears interdependent in many systems, but "who's on first" may differ. Genetic analysis in S. pombe has indicated that $\mathrm{H} 3-\mathrm{K} 9$ methylation precedes localization of Swi6, the HP1 homolog (for review, see Grewal and Elgin 2002). However, work by Schotta et al. (2002) demonstrates that the normal restriction of SU(VAR)3-9 (the major $\mathrm{H} 3-\mathrm{K} 9$ methyl transferase) and $\mathrm{H} 3-\mathrm{mK} 9$ to heterochromatic domains is dependent upon HP1 in Drosophila. Loss of HP1 results in mislocalization (presence throughout the euchromatic arms) of SU(VAR)3-9; loss of SU(VAR)3-9 results in a loss of HP1 in the pericentric heterochromatin, but a general delocalization is not observed. One can suggest that siRNA targeting of HP1 may be the initial event in heterochromatin formation, although histone modification is likely to be essential to generate a stable chromatin state. Both alternatives (HP1 on first vs. H3-K9 methylation first) appear plausible, and are indicated as possible routes to heterochromatin formation in Figure 6. The exciting challenge for the next several years is to determine which pathways are the correct ones and which players have lead roles. 


\section{ACKNOWLEDGMENTS}

We thank Michael Grunstein (UCLA) for making antibodies directed against modified histones available, and Gabriella Farkas (Washington University) for assistance in the preparation of figures. This work was supported by a grant from NIGMS, NIH, to S.C.R.E. (GM068388).

\section{REFERENCES}

Aravin A.A., Lagos-Quintana M., Yalcin A., Zavolan M., Marks D., Snyder B., Gaasterland T., Meyer J., and Tuschl T. 2003. The small RNA profile during Drosophila melanogaster development. Dev. Cell 5: 337.

Barigozzi C., Dolfini S., Fracacaro M., Rezzonico-Raimondi G., and Tiepolo L. 1966. In vitro study of the DNA replication patterns of somatic chromosomes of Drosophila melanogaster. Exp. Cell Res. 43: 231.

Berger S. 2002. Histone modifications in transcriptional regulation. Curr. Opin. Genet. Dev. 12: 142.

Bridges C.B. 1935. The mutants and linkage data of chromosome four of Drosophila melanogaster. Biol. Zh. 4: 401.

Crydernan D.E., Tang H., Gilmour D.S., and Wallrath L.L. 1999a. Heterochromatic silencing of Drosophila heat shock genes acts at the level of promoter potentiation. Nucleic Acids Res. 27: 3364.

Cryderman D.E., Morris E.J., Biessmann H., Elgin S.C., and Wallrath L.L. 1999b. Silencing at Drosophila telomeres: Nuclear organization and chromatin structure play critical roles. EMBO J. 18: 3724.

Eissenberg J.C. and Elgin S.C.R. 2000. The HP1 protein family: Getting a grip on chromatin. Curr. Opin. Genet. Dev. 10: 204.

Eissenberg J.C., James T.C., Foster-Hartnett D.M., Hartnett T., Ngan V., and Elgin S.C.R. 1990. A mutation in a heterochromatin-specific chromosomal protein is associated with suppression of position effect variegation in Drosophila melanogaster. Proc. Natl. Acad. Sci. 87: 9923.

FlyBase Consortium. 2003. The FlyBase database of the Drosophila genome projects and community literature (http://flybase.org/[release 3]). Nucleic Acids Res. 31: 172.

Gasser S.M. 2001. Positions of potential: Nuclear organization and gene expression. Cell 104: 639.

Grewal S. and Elgin S.C.R. 2002. Heterochromatin: New possibilities for the inheritance of structure. Curr. Opin. Genet. Dev. 12: 178.

Hall I.H., Shankaranarayana G.D., Noma K., Ayoub N., Cohen A., and Grewal S.I. 2002. Establishment and maintenance of a heterochromatin domain. Science 297: 2232.

Hannon G. 2002. RNA interference. Nature 418: 244.

Hoskins R.A., Smith C.D., Carlson J.W., Carvalho A.B., Halpern A., Kaminker J.S., Kennedy C., Mungall C.J., Sullivan B.A., Sutton G.G., Yasuhara J.C., Wakimoto B.T., Myers E.W., Celniker S.E., Rubin G.M., and Karpen G.H. 2002. Heterochromatic sequences in a Drosophila whole-genome shotgun assembly. Genome Biol. 3: RESEARCH0085.

James T.C., Eissenberg J.C., Craig C., Dietrich V., Hobson A., and Elgin S.C.R. 1989. Distribution patterns of HP1, a heterochromatin-associated nonhistone chromosomal protein of Drosophila. Eur. J. Cell Biol. 50: 170.

Kaminker J., Bergman V., Kronmileer B., Carlson J., Sviskas R., Patel S., Frise E., Wheeler D.A., Lewis S., Rubin G.M., Ashburner M., and Celniker S. 2002. The transposable elements of the Drosophila melanogaster euchromatin: A genomic perspective (http://genomebiology.com/2002/3/12/research/ 0084). Genome Biology 3: RESEARCH0084.

Matzke M., Aufsatz W., Kanno T., Daxinger L., Papp I., Mette M.F., and Matzke A.J.M. 2004. Genetic analysis of RNA-mediated transcriptional gene silencing. Biochim. Biophys. Acta 1677: 129.

Maurer-Stroh S., Dickens N.J., Hughes-Davies L., Kouzarides T., Eisenhaber F., Ponting C.P. 2003. The Tudor domain 'Royal Family': Tudor, plant Agenet, Chromo, PWWP and MBT domains. Trends Biochem. Sci. 28: 69.

Pal-Bhadra M., Leibovitch B.A., Gandhi S.G., Rao M., Bhadra U., Birchler J.A., and Elgin S.C.R. 2004. Heterochromatic silencing and HP1 localization in Drosophila are dependent on the RNAi machinery. Science 303: 669.

Schotta G., Ebert A., Krauss V., Fischer A., Hoffmann J., Rea S., Jenuwein T., Dorn R., and Reuter G. 2002. Central role of Drosophila SU(VAR)3-9 in histone H3-K9 methylation and heterochromatic gene silencing. EMBO J. 21: 1121.

Schramke V. and Allshire R. 2004. Those interfering little RNAs! Silencing and eliminating chromatin. Curr. Opin. Genet. Dev. 14: 174.

Suka N., Suka Y., Carmen A.A., Wu J., and Grunstein M. 2001. Highly specific antibodies determine histone acetylation site usage in yeast heterochromatin and euchromatin. Mol. Cell 8: 473.

Sun F.-L., Cuaycong M.H., and Elgin S.C.R. 2001. Long-range nucleosome ordering is associated with gene silencing in Drosophila melanogaster pericentric heterochromatin. Mol. Cell. Biol. 21: 2867.

Sun F.-L., Cuaycong M.H., Craig C.A., Wallrath L.L., Locke J., and Elgin S.C.R. 2000. The fourth chromosome of Drosophila melanogaster: Interspersed euchromatic and heterochromatic domains. Proc. Natl. Acad. Sci. 97: 5340.

Sun F.-L., Haynes K., Simpson C.L., Lee S.D., Collins L., Wuller J., Eissenberg J.C., and Elgin S.C.R. 2004. Cis-acting determinants of heterochromatin formation on Drosophila melanogaster chromosome four. Mol. Cell. Biol. 24: 18.

Verdel A., Jia S., Gerber S., Sugiyama T., Gygi S., Grewal S.I., and Moazed D. 2004. RNAi-mediated targeting of heterochromatin by the RITS complex. Science 303: 672 .

Volpe T.A., Kidner C., Hall I.M., Teng G., Grewal S.I., and Martienssen R.A. 2002. Regulation of heterochromatic silencing and histone H3 lysine-9 methylation by RNAi. Science 297: 1833.

Wallrath L.L. and Elgin S.C.R. 1995. Position effect variegation in Drosophila is associated with an altered chromatin structure. Genes Dev. 9: 1263. 


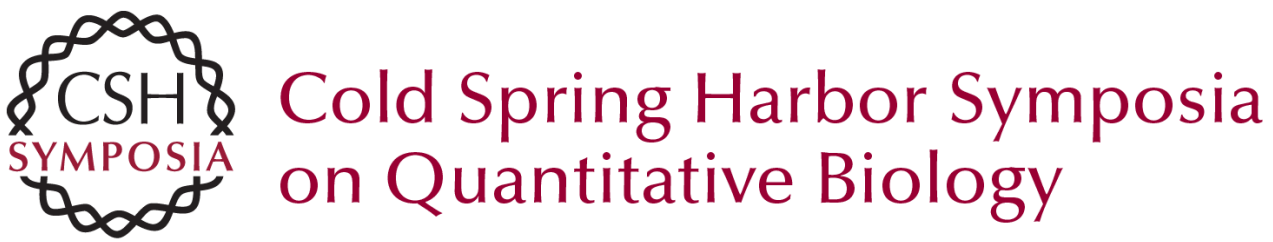

\title{
Analyzing Heterochromatin Formation Using Chromosome 4 of Drosophila melanogaster
}

\author{
K.A. HAYNES, B.A. LEIBOVITCH, S.H. RANGWALA, et al. \\ Cold Spring Harb Symp Quant Biol 2004 69: 267-272 \\ Access the most recent version at doi:10.1101/sqb.2004.69.267
}

References This article cites 28 articles, 10 of which can be accessed free at: http://symposium.cshlp.org/content/69/267.full.html\#ref-list-1

\section{License}

Email Alerting

Receive free email alerts when new articles cite this article - sign up in Service the box at the top right corner of the article or click here. 